Ю.Е. Стоян, А.В. Панкратов, Т.Е. Романова

Институт проблем машиностроения имени А.Н. Подгорного НАН Украиньл

\title{
УПАКОВКА МНОГОГРАННИКОВ В ВЫПУКЛЫЙ КОНТЕЙНЕР МИНИМАЛЬНОГО ОБЪЕМА
}

Рассматривается задача упаковки заданного набора произвольных многогранников в выпукльй контейнер минимального объема. Допускаются непрерывные вращзения и трансляции многогранников. Учитываются минимально допустимые расстояния между многогранниками и ограничения баланса. Построена математическая модель в виде задачи нелинейного программирования с использованием рһі-функций и квази рһі-функций. Предложен эффективный метод решения, который включает в себя быстрый алгоритм поиска допустимой стартовой точки и процедуру локальной оптимизации. Преимущество предложенного метода подтверждается результатами численных экспериментов.

Ключевые слова: упаковка, многогранники, непрерывное вращение, выпукльй контейнер, математическое моделирование, нелинейная оптимизация.

\section{Введение}

Задачи упаковки и раскроя ([1 - 3]) имеют очень широкий спектр применения, например, в биологии, минералогии, медицине, материаловедении, нанотехнологиях, робототехнике, системах распознавания образов, системах управления космическими аппаратами, а также в химической промышленности, энергетике, машиностроении, судостроении, авиастроении, гражданском строительстве.

В данном исследовании рассматриваются задачи упаковки набора произвольных многогранников в выпуклый контейнер минимального объема.

Одно из интересных применений для этой задачи возникает при оптимизации аддитивного производства, часто упоминаемого как 3D печать. Технологии аддитивного производства создают объект посредством добавления тонкого слоя материала за один цикл, например, вовремя екструзии материала или спекания слоев порошкового материала. Упаковка объектов в один компактный паттерн может уменьшить время печати, улучшая использование производственных мощностей, и уменьшить необходимость в дополнительном поддерживающем материале.

Задача оптимальной упаковки многогранников является NP-сложной [4], и, как следствие, методологии решения, как правило, используют эвристики (см., например, [5]-[8]). В некоторых работах (см., например, [9]-[11]) строятся математические модели и применяются методы математического программирования.

В данном исследовании предлагается подход, основанный на математическом моделировании отношений между геометрическими объектами с использованием метода рhі-функций [12], который позволяет сформулировать задачу упаковки в виде задачи нелинейного программирования. Рассматривается задача упаковки в следующей постановке.

\section{Постановка задачи}

Контейнерь. Имеется выпуклый контейнер $\Omega=\Omega(\mathrm{p})$ с переменными метрическими характеристиками, $\Omega=\left\{(\mathrm{x}, \mathrm{y}, \mathrm{z}, \mathrm{p}) \in \mathrm{R}^{3}: \Psi(\mathrm{x}, \mathrm{y}, \mathrm{z}, \mathrm{p}) \geq 0\right\}$, заданный в неподвижной системе координат OXYZ, где $\Psi(\mathrm{x}, \mathrm{y}, \mathrm{z}, \mathrm{p})=\min \left\{\Psi_{\mathrm{s}}(\mathrm{x}, \mathrm{y}, \mathrm{z}, \mathrm{p}), \mathrm{s}=1, \ldots, \mathrm{n}_{\Omega}\right\}$, $\Psi_{\mathrm{s}}(\mathrm{x}, \mathrm{y}, \mathrm{z}, \mathrm{p})$ - дифференцируемые функции, $\mathrm{s}=1, \ldots, \mathrm{n}_{\Omega}$. В частности, рассматриваются следующие виды контейнеров:

$$
\begin{gathered}
\mathbf{B}=\left\{(\mathrm{x}, \mathrm{y}, \mathrm{z}, \mathrm{l}, \mathrm{w}, \mathrm{h}) \in \mathrm{R}^{3} \mid \min \{\mathrm{x}+1,-\mathrm{x}+1,\right. \\
\mathrm{y}+\mathrm{w},-\mathrm{y}+\mathrm{w}, \mathrm{z}+\mathrm{h},-\mathrm{z}+\mathrm{h}\} \geq 0\}-
\end{gathered}
$$

кубоид с переменными размерами $1, \mathrm{w}$ и $\mathrm{h}, \mathrm{p}=(\mathrm{l}, \mathrm{w}, \mathrm{h})$;

$$
\mathbf{S}=\left\{(\mathrm{x}, \mathrm{y}, \mathrm{z}, \mathrm{r}) \in \mathbb{R}^{3} \mid \mathrm{r}^{2}-\mathrm{x}^{2}-\mathrm{y}^{2}-\mathrm{z}^{2} \geq 0\right\}-
$$

шар с переменным радиусом r, $\mathrm{p}=(\mathrm{r})$;

$$
\mathbf{E}=\left\{(\mathrm{x}, \mathrm{y}, \mathrm{z}, \lambda) \in \mathbb{R}^{3} \mid \lambda^{2}-\frac{\mathrm{x}^{2}}{\mathrm{a}^{2}}-\frac{\mathrm{y}^{2}}{\mathrm{~b}^{2}}-\frac{\mathrm{z}^{2}}{\mathrm{c}^{2}} \geq 0\right\}-
$$

эллипсоид с полуосями $\lambda \mathrm{a}, \lambda \mathrm{b}, \lambda \mathrm{c}, \mathrm{p}=(\lambda), \lambda-$ переменный коэффициент гомотетии, $\lambda=1$ соответствует исходным размерам контейнера;

$$
\begin{gathered}
\mathbf{C}=\left\{(\mathrm{x}, \mathrm{y}, \mathrm{z}, \lambda) \in \mathbb{R}^{3} \mid \min \left\{(\lambda \mathrm{r})^{2}-\mathrm{x}^{2}-\mathrm{y}^{2},\right.\right. \\
-\mathrm{z}+\lambda \mathrm{h}, \mathrm{z}+\lambda \mathrm{h}\} \geq 0\}-
\end{gathered}
$$

цилиндр высоты $\lambda \mathrm{h}$ и основанием радиуса $\lambda \mathrm{r}, \lambda-$ переменный коэффициент гомотетии, $\mathrm{p}=(\lambda), \lambda=1$ соответствует исходным размерам контейнера;

$$
\boldsymbol{\Lambda}=\left\{(\mathrm{x}, \mathrm{y}, \mathrm{z}) \in \mathrm{R}^{3} \mid \min _{\mathrm{s}=1,2} \Psi_{\mathrm{s}}(\mathrm{x}, \mathrm{y}, \mathrm{z}, \mathrm{r}) \geq 0\right\},-
$$

область, образованная непустым пересечением двух шаров с центрами в точках $\left(\mathrm{x}_{\mathrm{s}}, \mathrm{y}_{\mathrm{s}}, \mathrm{z}_{\mathrm{s}}\right)$ переменного радиуса $\mathrm{r}_{\mathrm{s}}=\mathrm{r}, \mathrm{p}=(\mathrm{r})$, где

$$
\Psi_{\mathrm{s}}(\mathrm{x}, \mathrm{y}, \mathrm{z}, \mathrm{r})=\mathrm{r}^{2}-\left(\mathrm{x}-\mathrm{x}_{\mathrm{s}}\right)^{2}-\left(\mathrm{y}-\mathrm{y}_{\mathrm{s}}\right)^{2}-\left(\mathrm{y}-\mathrm{z}_{\mathrm{s}}\right)^{2},
$$


$\mathrm{s}=1,2$. Полагаем, что $\left(\mathrm{x}_{1}=\mathrm{a}, \mathrm{y}_{1}=0, \mathrm{z}_{1}=0\right)$ $\left(\mathrm{x}_{2}=-\mathrm{a}, \mathrm{y}_{2}=0, \mathrm{z}_{2}=0\right)$, тогда

$$
\begin{aligned}
& \Psi_{1}(\mathrm{x}, \mathrm{y}, \mathrm{z}, \mathrm{r})=\mathrm{r}^{2}-(\mathrm{x}-\mathrm{a})^{2}-\mathrm{y}^{2}-\mathrm{z}^{2}, \\
& \Psi_{2}(\mathrm{x}, \mathrm{y}, \mathrm{z}, \mathrm{r})=\mathrm{r}^{2}-(\mathrm{x}+\mathrm{a})^{2}-\mathrm{y}^{2}-\mathrm{z}^{2} .
\end{aligned}
$$

Отметим, что каждая из метрических характеристик контейнера $\Omega$ может быть переменной сама по себе, например a,b,c - для эллипсоида или $\mathrm{r}, \mathrm{h}$ - для цилиндра.

Контейнер $\Omega$ имеет фиксированные параметры размещения $(0,0,0)$ в глобальной системе координат.

Объекты. Пусть $\{1,2, \ldots, \mathrm{N}\}=\mathrm{J}_{\mathrm{N}}$ и набор многогранников $\mathbb{Q}_{\mathrm{q}}, \mathrm{q} \in \mathrm{J}_{\mathrm{N}}$ заданы.

В целях данной работы предположим, что масса $\mathrm{M}_{\mathrm{q}}$ и центр массы $\mathrm{c}_{\mathrm{q}}$ каждого многогранника $\mathbb{Q}_{\mathrm{q}}$ известны.

С каждым из многогранников $\mathbb{Q}_{q}$ связана его локальная система координат с центром в $\mathrm{v}_{\mathrm{q}}$. Каждый невыпуклый многогранник $\mathbb{Q}_{\mathrm{q}}$ представлен в виде объединения выпуклых многогранников $\mathrm{K}_{\mathrm{j}}^{\mathrm{q}}$, $\mathrm{j}=1, \ldots, \mathrm{n}_{\mathrm{q}}$. Каждый выпуклый многогранник $\mathrm{K}_{\mathrm{j}}^{\mathrm{q}}$ определяется его вершинами $\mathrm{p}_{\mathrm{s}}^{\mathrm{qj}}, \mathrm{s}=1, \ldots ., \mathrm{m}_{\mathrm{j}}^{\mathrm{q}}$, в системе координат невыпуклого многогранника $\mathbb{Q}_{\mathrm{q}}$.

В пределах данного исследования полагаем что $\mathbb{Q}_{\mathrm{q}}=\bigcup_{\mathrm{j}=1}^{\mathrm{n}_{\mathrm{q}}} \mathrm{K}_{\mathrm{j}}^{\mathrm{q}}$ - известно. Не теряя общности, полагаем, также, что центр $\mathrm{v}_{\mathrm{q}}$ многогранника $\mathbb{Q}_{\mathrm{q}}$ совпадает с центром описанной вокруг него сферы $\mathrm{S}_{\mathrm{q}}$ радиуса $\mathrm{r}_{\mathrm{q}}$. Для построения описанной вокруг многогранника сферы используется быстрый алгоритм, предложенный в работе [13], который вычисляет наименьшую охватывающую сферу для набора точек (https://github.com/hbf/miniball).

Положение и ориентация каждого многогранника $\mathbb{Q}$ определяется вектором $\mathrm{u}=(\mathrm{v}, \theta)$ его переменных параметров размещения. Здесь $\mathrm{v}=(\mathrm{x}, \mathrm{y}, \mathrm{z}) \quad$ вектор трансляции, $\theta=\left(\theta^{1}, \theta^{2}, \theta^{3}\right)-$ вектор угловых параметров, $\theta^{1}, \theta^{2}, \theta^{3}$ углы Эйлера.

Многогранник, повернутый на $\theta$ и транслированный на вектор $\mathrm{v}$ обозначается через $\mathbb{Q}(\mathrm{u})=\left\{\mathrm{p} \in \mathrm{R}^{3}: \mathrm{p}=\mathrm{v}+\mathrm{M}(\theta) \cdot \mathrm{p}^{0}, \mathrm{p}^{0} \in \mathbb{Q}^{0}\right\}, \quad$ где $\mathrm{u}=(\mathrm{v}, \quad \theta), \quad \mathbb{Q}^{0} \quad-\quad$ неповернутый и нетранслированный многогранник $\mathrm{M}(\theta)=\mathrm{M}\left(\theta^{1}, \theta^{2}, \theta^{3}\right)-$ матрица поворота.
Между каждой парой многогранников $\mathbb{Q}_{\mathrm{q}}$ и $\mathbb{Q}_{\mathrm{g}}, \mathrm{q}<\mathrm{g} \in \mathrm{J}_{\mathrm{N}}$, могут быть заданы минимально допустимые расстояния, также как и между многогранником $\mathbb{Q}_{\mathrm{q}}, \mathrm{q} \in \mathrm{I}_{\mathrm{N}}$, и границей контейнера $\Omega$.

Ограничения баланса могут быть учтены как минимальное отклонение механической системы (контейнер с размещенными в нем объектами) от заданной точки. Рассматриваются различные типы функций цели, которые зависят от параметров размещения многогранников и переменных метрических характеристик контейнера $\Omega$. Отметим, что любая метрическая характеристика контейнера $\Omega$ может быть переменной либо фиксированной.

Задача упаковки многогранников может быть сформулирована следующим образом:

Упаковать набор многогранников $\mathbb{Q}_{\mathrm{q}}, \mathrm{q} \in \mathrm{J}_{\mathrm{N}}$, внутри выпуклого контейнера $\Omega$, учитывая геометрические и механические ограничения, так, чтобы функция цели достигала экстремума.

\section{Моделирование ограничений размещения}

В этом разделе описывается методология моделирования ограничений включения, непересечения и ограничений на минимально допустимые расстояния. Рассмотрим ограничения размещения, которые встречаются в задаче упаковки многогранников.

Пусть $\rho_{\mathrm{qg}}>0$ - минимально допустимое расстояние между парой многогранников $\mathbb{Q}_{\mathrm{q}}$ и $\mathbb{Q}_{\mathrm{g}}$ и $\rho_{\mathrm{q}}>0$ - минимально допустимое расстояние между многогранником $\mathbb{Q}_{\mathrm{q}}$ и объектом $\Omega^{*}$.

Сформулируем ограничения размещения следующим образом:

Ограничения непересечения

$$
\operatorname{int} \mathbb{Q}_{\mathrm{q}} \cap \text { int } \mathbb{Q}_{\mathrm{g}}=\varnothing, \mathrm{q}<\mathrm{g} \in \mathrm{J}_{\mathrm{N}} \cdot
$$

Ограничения включения $\mathbb{Q}_{\mathrm{q}} \subset \Omega \Leftrightarrow \operatorname{int} \mathbb{Q}_{\mathrm{q}} \cap \Omega^{*}=\varnothing, \mathrm{q} \in \mathrm{J}_{\mathrm{N}}, \Omega^{*}=\mathrm{R}^{3} \backslash \operatorname{int} \Omega$.

Ограничения на минимально допустимые расстояния между многогранниками

$$
\operatorname{dist}\left(\mathbb{Q}_{\mathrm{q}}, \mathbb{Q}_{\mathrm{g}}\right)=\min _{\mathrm{a} \in \mathbb{Q}_{\mathrm{q}}, \mathrm{b} \in \mathbb{Q}_{\mathrm{g}}} \mathrm{d}(\mathrm{a}, \mathrm{b}) \geq \rho_{\mathrm{qg}},
$$

где $\mathrm{d}(\mathrm{a}, \mathrm{b})$ - евклидово расстояние между $\mathrm{a}, \mathrm{b} \in \mathrm{R}^{3}$.

Ограничения на минимально допустимые расстояния между многогранником и объектом $\Omega^{*}$

$$
\operatorname{dist}\left(\mathbb{Q}_{\mathrm{q}}, \Omega^{*}\right) \geq \rho_{\mathrm{q}}, \mathrm{q} \in \mathrm{J}_{\mathrm{N}} .
$$

Отметим, что, если допустимые расстояния не заданы, то имеем дело с ограничениями непересечения, int $\mathbb{Q}_{\mathrm{q}} \cap \operatorname{int} \mathbb{Q}_{\mathrm{g}}=\varnothing$, и ограничениями включения, $\mathbb{Q}_{\mathrm{q}} \subset \Omega \Leftrightarrow \operatorname{int} \mathbb{Q}_{\mathrm{q}} \cap \Omega^{*}$. 
С целью аналитического описания ограничений размещения, перечисленных выше, используется метод рhі-функций [12].

Ограничения непересечения. Для построения квази рhі-функций и псевдонормализованных квази phi-функций для двух невыпуклых многогранников используются квази рhі-функции и псевдонормализованные квази рhі-функции для каждой пары выпуклых многогранников, которые формируют исходные невыпуклые многогранники.

Прежде всего, рассмотрим квази рhі-функцию для пары выпуклых многогранников.

Пусть $\mathrm{A}\left(\mathrm{u}_{\mathrm{A}}\right)$ и $\mathrm{B}\left(\mathrm{u}_{\mathrm{B}}\right)$ - два выпуклых многогранника, заданных собственными вершинами $\mathrm{p}_{\mathrm{s}}^{\mathrm{A}}$, $\mathrm{s}=1, \ldots, \mathrm{m}_{\mathrm{A}}$, и $\mathrm{p}_{\mathrm{s}}^{\mathrm{B}}, \mathrm{s}=1, \ldots, \mathrm{m}_{\mathrm{B}}$.

Квази рhі-функция $\Phi^{\prime A B}\left(u_{A}, u_{B}, u^{\prime}=u_{P}\right)$ для выпуклых многогранников $\mathrm{A}\left(\mathrm{u}_{\mathrm{A}}\right)$ и $\mathrm{B}\left(\mathrm{u}_{\mathrm{B}}\right)$ может быть определена следующим образом:

$$
\begin{gathered}
\Phi^{\prime \mathrm{AB}}\left(\mathrm{u}_{\mathrm{A}}, \mathrm{u}_{\mathrm{B}}, \mathrm{u}^{\prime}=\mathrm{u}_{\mathrm{P}}\right)= \\
=\min \left\{\Phi^{\mathrm{AP}}\left(\mathrm{u}_{\mathrm{A}}, \mathrm{u}_{\mathrm{P}}\right), \Phi^{\mathrm{BP}^{*}}\left(\mathrm{u}_{\mathrm{B}}, \mathrm{u}_{\mathrm{P}}\right)\right\}
\end{gathered},
$$

где $\Phi^{\mathrm{AP}}\left(\mathrm{u}_{\mathrm{A}}, \mathrm{u}_{\mathrm{P}}\right)$ - phi-функция для $\mathrm{A}\left(\mathrm{u}_{\mathrm{A}}\right)$ и $\mathrm{P}\left(\mathrm{u}_{\mathrm{P}}\right)$, $\Phi^{\mathrm{BP}}\left(\mathrm{u}_{\mathrm{B}}, \mathrm{u}_{\mathrm{P}}\right)$ - phi-функция для $\mathrm{B}\left(\mathrm{u}_{\mathrm{B}}\right)$ и $\mathrm{P}^{*}\left(\mathrm{u}_{\mathrm{P}}\right)==\mathrm{R}^{3} \backslash \operatorname{int} \mathrm{P}\left(\mathrm{u}_{\mathrm{P}}\right)$,

$\mathrm{P}\left(\mathrm{u}_{\mathrm{P}}\right)=\left\{(\mathrm{x}, \mathrm{y}, \mathrm{z}): \psi_{\mathrm{P}}=\alpha \cdot \mathrm{x}+\beta \cdot \mathrm{y}+\gamma \cdot \mathrm{z}+\mu_{\mathrm{P}} \leq 0\right\}$,

$\alpha=\sin \theta_{\mathrm{P}}^{1} \sin \theta_{\mathrm{P}}^{2}, \beta=-\cos \theta_{\mathrm{P}}^{1} \sin \theta_{\mathrm{P}}^{2}, \gamma=\cos \theta_{\mathrm{P}}^{2}$,

$\theta_{\mathrm{P}}^{1}$ и $\theta_{\mathrm{P}}^{2}$ соответствующие переменные углы Ейлера (при $\left.\theta_{\mathrm{P}}^{3}=0\right), \mathrm{u}_{\mathrm{P}}=\left(\theta_{\mathrm{P}}^{1}, \theta_{\mathrm{P}}^{2}, \mu_{\mathrm{P}}\right)$ - вектор переменных параметров, которые определяют плоскость $\mathrm{L}_{\mathrm{AB}}=\left\{(\mathrm{x}, \mathrm{y}, \mathrm{z}): \psi_{\mathrm{P}}=0\right\}$ в трехмерном Евклидовом пространстве $\left(\alpha^{2}+\beta^{2}+\gamma^{2}=1\right)$,

$$
\begin{gathered}
\Phi^{\mathrm{AP}}\left(\mathrm{u}_{\mathrm{A}}, \mathrm{u}_{\mathrm{P}}\right)=\min _{1 \leq \mathrm{s} \leq \mathrm{m}_{\mathrm{A}}} \psi_{\mathrm{P}}\left(\mathrm{p}_{\mathrm{s}}^{\mathrm{A}}\right), \\
\Phi^{\mathrm{BP}}\left(\mathrm{u}_{\mathrm{B}}, \mathrm{u}_{\mathrm{P}}\right)=\min _{1 \leq \mathrm{s} \leq \mathrm{m}_{\mathrm{B}}}\left(-\psi_{\mathrm{P}}\left(\mathrm{p}_{\mathrm{s}}^{\mathrm{B}}\right)\right) .
\end{gathered}
$$

Как известно [14], если $\Phi^{\prime A B}\left(u_{\mathrm{A}}, \mathrm{u}_{\mathrm{B}}, \mathrm{u}_{\mathrm{P}}\right) \geq 0$ для некоторых $\mathrm{u}_{\mathrm{P}}$, тогда int $\mathrm{A}\left(\mathrm{u}_{\mathrm{A}}\right) \bigcap \operatorname{int} \mathrm{B}\left(\mathrm{u}_{\mathrm{B}}\right)=\varnothing$.

Пусть задано минимально допустимое расстояние $\rho_{\text {AB }}$ между двумя произвольными выпуклыми многогранниками $\mathrm{A}\left(\mathrm{u}_{\mathrm{A}}\right)$ и $\mathrm{B}\left(\mathrm{u}_{\mathrm{B}}\right)$. Для описания ограничения на допустимые расстояния, dist ( A, B ) $\geq \rho_{\mathrm{AB}}$, используются псевдонормализованные квази phi-функции для выпуклых многогранников $\mathrm{A}\left(\mathrm{u}_{\mathrm{A}}\right)$ и $\mathrm{B}\left(\mathrm{u}_{\mathrm{B}}\right)$, полученные следующим образом

$$
\begin{aligned}
& \widehat{\Phi}^{\prime \mathrm{AB}}\left(\mathrm{u}_{\mathrm{A}}, \mathrm{u}_{\mathrm{B}}, \mathrm{u}_{\mathrm{P}}\right)=\Phi^{\prime \mathrm{AB}}\left(\mathrm{u}_{\mathrm{A}}, \mathrm{u}_{\mathrm{B}}, \mathrm{u}_{\mathrm{P}}\right)-0.5 \rho_{\mathrm{AB}} \\
& \text { Заметим, что } \\
& \max _{\Phi^{\prime}} \mathrm{AB}\left(\mathrm{u}_{\mathrm{A}}, \mathrm{u}_{\mathrm{B}}, \mathrm{u}_{\mathrm{P}}\right) \geq 0 \Leftrightarrow \operatorname{dist}(\mathrm{A}, \mathrm{B}) \geq \rho_{\mathrm{AB}} .
\end{aligned}
$$

Прежде, чем определить квази рhі-функции и псевдонормализованные квази рhі-функции для пары невыпуклых многогранников, представим заданный набор выпуклых многогранников, $\mathrm{K}_{\mathrm{j}}^{\mathrm{q}}$, $\mathrm{j}=1, \ldots, \mathrm{n}_{\mathrm{q}}, \mathrm{q} \in \mathrm{J}_{\mathrm{N}}$, в виде множества, соостоящего из $\mathrm{n}=\sum_{\mathrm{q}=1}^{\mathrm{N}} \mathrm{n}_{\mathrm{q}}$ выпуклых многогранников $\mathrm{K}_{\mathrm{i}}$, $\mathrm{i} \in\{1,2, \ldots, \mathrm{n}\}=\mathrm{I}_{\mathrm{n}}$, используя следующее правило: $\mathrm{K}_{\mathrm{j}}^{\mathrm{q}} \rightarrow \mathrm{K}_{\mathrm{i}}, \mathrm{i}=\sum_{\mathrm{l}=0}^{\mathrm{q}-1} \mathrm{n}_{1}+\mathrm{j}, \mathrm{j}=1, \ldots, \mathrm{n}_{\mathrm{q}}, \mathrm{q} \in \mathrm{J}_{\mathrm{N}}$, при условии, что $\mathrm{n}_{0}=0$.

Введем вектор $\mathbf{a}=\left(\mathrm{a}_{1},,,, \mathrm{a}_{\mathrm{n}}\right), \mathrm{a}_{\mathrm{i}} \in \mathrm{J}_{\mathrm{N}}, \quad$ где $\mathrm{a}_{\mathrm{i}}=\mathrm{q}, \quad$ если $\mathrm{K}_{\mathrm{i}} \subset \mathbb{Q}_{\mathrm{q}}, \quad \mathrm{q} \in \mathrm{J}_{\mathrm{N}} \cdot$ Пусть $\mathrm{I}_{\mathrm{n}}=\mathrm{I}^{1} \cup \mathrm{I}^{2} \cup \ldots \mathrm{I}^{\mathrm{N}}$ - упорядоченное разбиение $\mathrm{I}_{\mathrm{n}}$, где $\mathrm{I}^{\mathrm{q}}=\left\{\mathrm{i} \in \mathrm{I}_{\mathrm{n}}, \mathrm{a}_{\mathrm{i}}=\mathrm{q}\right\},\left|\mathrm{I}^{\mathrm{q}}\right|=\mathrm{n}_{\mathrm{q}}, \mathrm{q} \in \mathrm{J}_{\mathrm{N}}$. Пусть $\mathbb{Q}_{\mathrm{q}}\left(\mathrm{u}_{\mathrm{q}}\right)=\bigcup_{\mathrm{i} \in \mathrm{I}^{\mathrm{q}}} \mathrm{K}_{\mathrm{i}}\left(\mathrm{u}_{\mathrm{q}}\right)$ и $\mathbb{Q}_{\mathrm{g}}\left(\mathrm{u}_{\mathrm{g}}\right)=\bigcup_{\mathrm{j} \in \mathrm{I}^{\mathrm{g}}} \mathrm{K}_{\mathrm{j}}\left(\mathrm{u}_{\mathrm{g}}\right)$ - невыпуклые многогранники и $\mathrm{q} \neq \mathrm{g}$. Тогда квази phiфункция для невыпуклых многогранников $\mathbb{Q}_{\mathrm{q}}\left(\mathrm{u}_{\mathrm{q}}\right)$ и $\mathbb{Q}_{\mathrm{g}}\left(\mathrm{u}_{\mathrm{g}}\right)$ может быть определена в виде:

$$
\begin{gathered}
\widehat{\Phi}_{\mathrm{qg}}^{\prime}\left(\mathrm{u}_{\mathrm{q}}, \mathrm{u}_{\mathrm{g}}, \mathrm{u}_{\mathrm{qg}}\right)= \\
=\min \left\{\widehat{\Phi}_{\mathrm{ij}}^{\prime}\left(\mathrm{u}_{\mathrm{q}}, \mathrm{u}_{\mathrm{g}}, \mathrm{u}_{\mathrm{ij}}^{\prime}\right), \mathrm{i} \in \mathrm{I}^{\mathrm{q}}, \mathrm{j} \in \mathrm{I}^{\mathrm{g}}\right\}
\end{gathered},
$$

где $\widehat{\Phi}_{\mathrm{ij}}^{\prime}\left(\mathrm{u}_{\mathrm{q}}, \mathrm{u}_{\mathrm{g}}, \mathrm{u}_{\mathrm{ij}}^{\prime}\right)$ - псевдонормализованная квази phi-функция и $\mathrm{u}_{\mathrm{ij}}^{\prime}$ - вектор вспомогательных переменных для выпуклых многогранников $\mathrm{K}_{\mathrm{i}}\left(\mathrm{u}_{\mathrm{q}}\right)$ и $\mathrm{K}_{\mathrm{j}}\left(\mathrm{u}_{\mathrm{g}}\right), \mathrm{i} \in \mathrm{I}^{\mathrm{q}}, \mathrm{j} \in \mathrm{I}^{\mathrm{g}}, \mathrm{u}_{\mathrm{qg}}=\left(\mathrm{u}_{\mathrm{ij}}^{\prime}, \mathrm{i} \in \mathrm{I}^{\mathrm{q}}, \mathrm{j} \in \mathrm{I}^{\mathrm{g}}\right)$.

Ограничения включения. Псевдонормализованная рhі-функция для невыпуклого многогранника $\mathbb{Q}_{\mathrm{q}}\left(\mathrm{u}_{\mathrm{q}}\right)$ и объекта $\Omega^{*}$ может быть определена в виде

$$
\widehat{\Phi}_{\mathrm{q}}\left(\mathrm{u}_{\mathrm{q}}\right)=\min \left\{\widehat{\Phi}_{\mathrm{i}}\left(\mathrm{u}_{\mathrm{q}}\right), \mathrm{i} \in \mathrm{I}^{\mathrm{q}}\right\}
$$

где $\widehat{\Phi}_{\mathrm{i}}\left(\mathrm{u}_{\mathrm{q}}\right)$ - псевдонормализованная рhі-функция для выпуклого многогранника $\mathrm{K}_{\mathrm{i}}\left(\mathrm{u}_{\mathrm{q}}\right)$ и $\Omega^{*}, \mathrm{i} \in \mathrm{I}^{\mathrm{q}}$. Заменяя каждую псевдонормализованную phiфункцию $\widehat{\Phi}_{\mathrm{i}}\left(\mathrm{u}_{\mathrm{q}}\right)$ в (4) на phi-функцию $\Phi_{\mathrm{i}}\left(\mathrm{u}_{\mathrm{q}}\right)$ для $\mathrm{i} \in \mathrm{I}^{\mathrm{q}}$, получаем рhі-функцию $\Phi_{\mathrm{q}}\left(\mathrm{u}_{\mathrm{q}}\right)$ для многогранника $\mathbb{Q}_{\mathrm{q}}\left(\mathrm{u}_{\mathrm{q}}\right)$ и объекта $\Omega^{*}$.

Для описания ограничений включения, $\mathrm{K}_{\mathrm{i}}\left(\mathrm{u}_{\mathrm{q}}\right) \subset \Omega \Leftrightarrow \operatorname{int} \mathrm{K}_{\mathrm{i}}\left(\mathrm{u}_{\mathrm{q}}\right) \cap \Omega^{*}=\varnothing, \quad$ используются phi-функции для выпуклого многогранника $\mathrm{K}_{\mathrm{i}}\left(\mathrm{u}_{\mathrm{q}}\right)$ и объекта $\Omega^{*}$ [12].

Пусть $\mathrm{K}_{\mathrm{i}}\left(\mathrm{u}_{\mathrm{q}}\right)$ - выпуклый многогранник, заданный в собственной локальной системе координат 
своими вершинами $\mathrm{p}_{\mathrm{k}}^{\mathrm{i}}, \quad \mathrm{k}=1, \ldots ., \mathrm{m}_{\mathrm{i}}, \quad$ где $\mathrm{p}_{\mathrm{k}}^{\mathrm{i}}=\left(\mathrm{p}_{\mathrm{xk}}^{\mathrm{i}}, \mathrm{p}_{\mathrm{yk}}^{\mathrm{i}}, \mathrm{p}_{\mathrm{zk}}^{\mathrm{i}}\right)$. Рhi-функция для $\mathrm{K}_{\mathrm{i}}\left(\mathrm{u}_{\mathrm{q}}\right)$ и объекта $\Omega^{*}$ имеет вид

$$
\begin{gathered}
\Phi_{\mathrm{i}}\left(\mathrm{u}_{\mathrm{q}}, \mathrm{p}\right)=\min \left\{\Psi_{\mathrm{s}}\left(\mathrm{u}_{\mathrm{q}}, \mathrm{p}\right), \mathrm{s}=1, \ldots, \mathrm{n}_{\Omega}\right\}, \\
\Psi_{\mathrm{s}}\left(\mathrm{u}_{\mathrm{q}}, \mathrm{p}\right)=\min \left\{\varphi_{\mathrm{s}}\left(\mathrm{p}_{\mathrm{k}}^{\mathrm{i}}\left(\mathrm{u}_{\mathrm{q}}\right), \mathrm{p}\right), \mathrm{k}=1, \ldots, \mathrm{m}_{\mathrm{i}}\right\} .
\end{gathered}
$$

В частности, используются следующие phiфункции для описания ограничений включения.

Рhi-функция для выпуклого многогранника $\mathrm{K}_{\mathrm{i}}\left(\mathrm{u}_{\mathrm{q}}\right)$ и объекта $\mathbf{S}^{*}=\mathbb{R}^{3} / \operatorname{int} \mathbf{S}$

$$
\begin{gathered}
\Phi^{\mathrm{K}_{\mathrm{i}} \mathrm{S}^{*}}\left(\mathrm{u}_{\mathrm{q}}, \mathrm{r}\right)=\min \left\{\varphi\left(\mathrm{p}_{\mathrm{k}}^{\mathrm{i}}\left(\mathrm{u}_{\mathrm{q}}\right), \mathrm{r}\right), \mathrm{k}=1, \ldots, \mathrm{m}_{\mathrm{i}}\right\}, \\
\varphi\left(\mathrm{p}_{\mathrm{k}}^{\mathrm{i}}\left(\mathrm{u}_{\mathrm{q}}\right), \mathrm{r}\right)= \\
=\mathrm{r}^{2}-\left(\mathrm{p}_{\mathrm{kx}}^{\mathrm{i}}\left(\mathrm{u}_{\mathrm{q}}\right)\right)^{2}-\left(\mathrm{p}_{\mathrm{ky}}^{\mathrm{i}}\left(\mathrm{u}_{\mathrm{q}}\right)\right)^{2}-\left(\mathrm{p}_{\mathrm{kz}}^{\mathrm{i}}\left(\mathrm{u}_{\mathrm{q}}\right)\right)^{2} .
\end{gathered}
$$

Рhі-функция для выпуклого многогранника $\mathrm{K}_{\mathrm{i}}\left(\mathrm{u}_{\mathrm{q}}\right)$ и объекта $\mathbf{E}^{*}=\mathbb{R}^{3} / \operatorname{int} \mathbf{E}$

$$
\begin{gathered}
\Phi^{\mathrm{K}_{\mathrm{i}} \mathbf{E}^{*}}\left(\mathrm{u}_{\mathrm{q}}, \lambda\right)=\min \left\{\varphi\left(\mathrm{p}_{\mathrm{k}}^{\mathrm{i}}\left(\mathrm{u}_{\mathrm{q}}\right), \lambda\right), \mathrm{k}=1, \ldots, \mathrm{m}_{\mathrm{i}}\right\}, \\
\varphi\left(\mathrm{p}_{\mathrm{k}}^{\mathrm{i}}\left(\mathrm{u}_{\mathrm{q}}\right), \lambda\right)= \\
=\lambda^{2}-\frac{\left(\mathrm{p}_{\mathrm{kx}}^{\mathrm{i}}\left(\mathrm{u}_{\mathrm{q}}\right)\right)^{2}}{\mathrm{a}^{2}}-\frac{\left(\mathrm{p}_{\mathrm{ky}}^{\mathrm{i}}\left(\mathrm{u}_{\mathrm{q}}\right)\right)^{2}}{\mathrm{~b}^{2}}-\frac{\left(\mathrm{p}_{\mathrm{kz}}^{\mathrm{i}}\left(\mathrm{u}_{\mathrm{q}}\right)\right)^{2}}{\mathrm{c}^{2}} .
\end{gathered}
$$

Рhі-функция для выпуклого многогранника $\mathrm{K}_{\mathrm{i}}\left(\mathrm{u}_{\mathrm{q}}\right)$ и объекта $\mathbf{B}^{*}=\mathbb{R}^{3} / \operatorname{int} \mathbf{B}$

$\Phi^{\mathrm{K}_{\mathrm{i}} \mathbf{B}^{*}}\left(\mathrm{u}_{\mathrm{q}}, 1, \mathrm{~h}, \mathrm{w}\right)=\min \left\{\Psi_{\mathrm{s}}\left(\mathrm{u}_{\mathrm{q}}, 1, \mathrm{~h}, \mathrm{w}\right), \mathrm{s}=1, \ldots, 6\right\}$,

$\Psi_{\mathrm{s}}\left(\mathrm{u}_{\mathrm{q}}, 1, \mathrm{~h}, \mathrm{w}\right)=\min \left\{\varphi_{\mathrm{ks}}^{\mathrm{i}}\left(\mathrm{u}_{\mathrm{q}}, 1, \mathrm{~h}, \mathrm{w}\right), \mathrm{k}=1, \ldots, \mathrm{m}_{\mathrm{i}}\right\}$,

$\varphi_{\mathrm{k} 1}^{\mathrm{i}}\left(\mathrm{u}_{\mathrm{q}}, 1, \mathrm{~h}, \mathrm{w}\right)=\mathrm{p}_{\mathrm{xk}}^{\mathrm{i}}\left(\mathrm{u}_{\mathrm{q}}\right)+1, \varphi_{\mathrm{k} 2}^{\mathrm{i}}\left(\mathrm{u}_{\mathrm{q}}\right)=-\mathrm{p}_{\mathrm{xk}}^{\mathrm{i}}\left(\mathrm{u}_{\mathrm{q}}\right)+1$,

$$
\begin{gathered}
\varphi_{\mathrm{k} 3}^{\mathrm{i}}\left(\mathrm{u}_{\mathrm{q}}, 1, \mathrm{~h}, \mathrm{w}\right)=\mathrm{p}_{\mathrm{yk}}^{\mathrm{i}}\left(\mathrm{u}_{\mathrm{q}}\right)+\mathrm{w}, \\
\varphi_{\mathrm{k} 4}^{\mathrm{i}}\left(\mathrm{u}_{\mathrm{q}}, 1, \mathrm{~h}, \mathrm{w}\right)=-\mathrm{p}_{\mathrm{yk}}^{\mathrm{i}}\left(\mathrm{u}_{\mathrm{q}}\right)+\mathrm{w}, \\
\varphi_{\mathrm{k} 5}^{\mathrm{i}}\left(\mathrm{u}_{\mathrm{q}}, 1, \mathrm{~h}, \mathrm{w}\right)=\mathrm{p}_{\mathrm{zk}}^{\mathrm{i}}\left(\mathrm{u}_{\mathrm{q}}\right)+\mathrm{h}, \\
\varphi_{\mathrm{k} 6}^{\mathrm{i}}\left(\mathrm{u}_{\mathrm{q}}, 1, \mathrm{~h}, \mathrm{w}\right)=-\mathrm{p}_{\mathrm{zk}}^{\mathrm{i}}\left(\mathrm{u}_{\mathrm{q}}\right)+\mathrm{h} .
\end{gathered}
$$

Рhi-функиия для выпуклого многогранника $\mathrm{K}_{\mathrm{i}}\left(\mathrm{u}_{\mathrm{q}}\right)$ и объекта $\mathbf{C}^{*}=\mathbb{R}^{3} / \operatorname{int} \mathbf{C}$

$$
\begin{gathered}
\Phi^{\mathrm{K}_{\mathrm{i}} \mathbf{c}^{*}}\left(\mathrm{u}_{\mathrm{q}}, \lambda\right)=\min \left\{\Psi_{\mathrm{s}}\left(\mathrm{u}_{\mathrm{q}}, \lambda\right), \mathrm{s}=1,2,3\right\}, \\
\Psi_{\mathrm{s}}\left(\mathrm{u}_{\mathrm{q}}, \lambda\right)=\min \left\{\varphi_{\mathrm{s}}\left(\mathrm{p}_{\mathrm{k}}^{\mathrm{i}}\left(\mathrm{u}_{\mathrm{q}}\right), \mathrm{r}\right), \mathrm{k}=1, \ldots, \mathrm{m}_{\mathrm{i}}\right\}, \\
\varphi_{1}\left(\mathrm{p}_{\mathrm{k}}^{\mathrm{i}}\left(\mathrm{u}_{\mathrm{q}}\right), \lambda\right)=(\lambda \mathrm{r})^{2}-\left(\mathrm{p}_{\mathrm{xk}}^{\mathrm{i}}\left(\mathrm{u}_{\mathrm{q}}\right)\right)^{2}-\left(\mathrm{p}_{\mathrm{yk}}^{\mathrm{i}}\left(\mathrm{u}_{\mathrm{q}}\right)\right)^{2}, \\
\varphi_{2}\left(\mathrm{p}_{\mathrm{k}}^{\mathrm{i}}\left(\mathrm{u}_{\mathrm{q}}\right), \lambda\right)=-\mathrm{p}_{\mathrm{zk}}^{\mathrm{i}}\left(\mathrm{u}_{\mathrm{q}}\right)+\lambda \mathrm{h}, \\
\varphi_{3}\left(\mathrm{p}_{\mathrm{k}}^{\mathrm{i}}\left(\mathrm{u}_{\mathrm{q}}\right), \lambda\right)=\mathrm{p}_{\mathrm{zk}}^{\mathrm{i}}\left(\mathrm{u}_{\mathrm{q}}\right)+\lambda \mathrm{h} .
\end{gathered}
$$

Phі-функция для выпуклого многогранника $\mathrm{K}_{\mathrm{i}}\left(\mathrm{u}_{\mathrm{q}}\right)$ и объекта $\boldsymbol{\Lambda}^{*}=\mathbb{R}^{3} / \operatorname{int} \boldsymbol{\Lambda}$

$$
\Phi^{\mathrm{K}_{\mathrm{i}} \Lambda^{*}}\left(\mathrm{u}_{\mathrm{q}}, \mathrm{r}\right)=\min \left\{\Psi_{1}\left(\mathrm{u}_{\mathrm{q}}, \mathrm{r}\right), \Psi_{2}\left(\mathrm{u}_{\mathrm{q}}, \mathrm{r}\right)\right\}
$$

$$
\begin{gathered}
\Psi_{1}\left(\mathrm{u}_{\mathrm{q}}, \mathrm{r}\right)=\min \left\{\varphi_{1}\left(\mathrm{p}_{\mathrm{k}}^{\mathrm{i}}\left(\mathrm{u}_{\mathrm{q}}\right), \mathrm{r}\right), \mathrm{k}=1, \ldots, \mathrm{m}_{\mathrm{i}}\right\}, \\
\varphi_{1}\left(\mathrm{p}_{\mathrm{k}}^{\mathrm{i}}\left(\mathrm{u}_{\mathrm{q}}\right), \mathrm{r}\right)= \\
=\mathrm{r}^{2}-\left(\mathrm{p}_{\mathrm{kx}}^{\mathrm{i}}\left(\mathrm{u}_{\mathrm{q}}\right)-\mathrm{a}\right)^{2}-\left(\mathrm{p}_{\mathrm{ky}}^{\mathrm{i}}\left(\mathrm{u}_{\mathrm{q}}\right)\right)^{2}-\left(\mathrm{p}_{\mathrm{kz}}^{\mathrm{i}}\left(\mathrm{u}_{\mathrm{q}}\right)\right)^{2} . \\
\Psi_{2}\left(\mathrm{u}_{\mathrm{q}}, \mathrm{r}\right)=\min \left\{\varphi_{2}\left(\mathrm{p}_{\mathrm{k}}^{\mathrm{i}}\left(\mathrm{u}_{\mathrm{q}}\right), \mathrm{r}\right), \mathrm{k}=1, \ldots, \mathrm{m}_{\mathrm{i}}\right\}, \\
\varphi_{2}\left(\mathrm{p}_{\mathrm{k}}^{\mathrm{i}}\left(\mathrm{u}_{\mathrm{q}}\right), \mathrm{r}\right)= \\
\mathrm{r}^{2}-\left(\mathrm{p}_{\mathrm{kx}}^{\mathrm{i}}\left(\mathrm{u}_{\mathrm{q}}\right)+\mathrm{a}\right)^{2}-\left(\mathrm{p}_{\mathrm{ky}}^{\mathrm{i}}\left(\mathrm{u}_{\mathrm{q}}\right)\right)^{2}-\left(\mathrm{p}_{\mathrm{kz}}^{\mathrm{i}}\left(\mathrm{u}_{\mathrm{q}}\right)\right)^{2} .
\end{gathered}
$$

Пусть задано минимально допустимое расстояние $\rho_{\mathrm{q}}>0$ между выпуклым многогранником $\mathrm{K}_{\mathrm{i}}\left(\mathrm{u}_{\mathrm{q}}\right)$ и объектом $\Omega^{*}$. Для описания ограничения на допустимые расстояния, dist $\left(\mathrm{K}_{\mathrm{i}}, \Omega^{*}\right) \geq \rho_{\mathrm{q}}$, используется псевдонормализованная рhі-функция для выпуклого многогранника $\mathrm{K}_{\mathrm{i}}\left(\mathrm{u}_{\mathrm{q}}\right)$ и объекта $\Omega^{*}$ вида

$$
\widehat{\Phi}_{\mathrm{i}}\left(\mathrm{u}_{\mathrm{q}}\right)=\Phi_{\mathrm{i}}\left(\mathrm{u}_{\mathrm{q}}\right)-\rho_{\mathrm{q}} \text {. }
$$

\section{Математическая модель}

Вектор $\mathrm{u} \in \mathrm{R}^{\sigma}$ всех переменных может быть описан следующим образом: $u=(\varsigma, \tau) \in \mathrm{R}^{\sigma}$, где $\varsigma=\left(\mathrm{p}, \mathrm{u}_{1}, \mathrm{u}_{2}, \ldots, \mathrm{u}_{\mathrm{N}}\right), \mathrm{p}$ - вектор переменных метрических характеристик заданного контейнера $\Omega$ и $\mathrm{u}_{\mathrm{a}_{\mathrm{i}}}=\left(\mathrm{v}_{\mathrm{a}_{\mathrm{i}}}, \theta_{\mathrm{a}_{\mathrm{i}}}\right)=\left(\mathrm{x}_{\mathrm{a}_{\mathrm{i}}}, \mathrm{y}_{\mathrm{a}_{\mathrm{i}}}, \mathrm{z}_{\mathrm{a}_{\mathrm{i}}}, \theta_{\mathrm{a}_{\mathrm{i}}}^{1}, \theta_{\mathrm{a}_{\mathrm{i}}}^{2}, \theta_{\mathrm{a}_{\mathrm{i}}}^{3}\right)$ - вектор параметров размещения $\mathrm{K}_{\mathrm{i}}, \quad \mathrm{i} \in \mathrm{I}_{\mathrm{n}}$, индекс $\mathrm{a}_{\mathrm{i}} \in\{1,2, \ldots, \mathrm{N}\}$ - компонента “склеивающего” вектора $\mathbf{a}$. Здесь $\tau=\left(\mathrm{u}_{\mathrm{P}}^{1}, \ldots, \mathrm{u}_{\mathrm{P}}^{\mathrm{m}}\right)$ - вектор всех вспомогательных переменных, $u_{\mathrm{P}}^{\mathrm{s}}=\left(\theta_{\mathrm{P}}^{1 \mathrm{~s}}, \theta_{\mathrm{P}}^{2 \mathrm{~s}}, \mu_{\mathrm{P}}^{\mathrm{s}}\right)-$ вектор дополнительных переменных для $s$-ой пары выпуклых многогранников определенных в (1), s = 1,.., m, $\mathrm{m}=\operatorname{card}(\Xi)$,

$$
\Xi=\left\{(\mathrm{i}, \mathrm{j}), \mathrm{a}_{\mathrm{i}} \neq \mathrm{a}_{\mathrm{j}}, \mathrm{i}<\mathrm{j} \in \mathrm{I}_{\mathrm{n}}\right\},
$$

$\sigma=\mathrm{p}+6 \mathrm{~N}+3 \mathrm{~m}-$ размерность задачи.

Математическая модель задачи упаковки многогранников может быть сформулирована в следующем виде

$$
\begin{gathered}
\min _{u \in \mathrm{W} \subset \mathrm{R}^{\sigma}} \mathrm{F}(\mathrm{u}), \\
\mathrm{W}=\left\{\mathrm{u} \in \mathrm{R}^{\sigma}: \widehat{\Phi}_{\mathrm{ij}^{\prime}}^{\prime}\left(\mathrm{u}_{\mathrm{a}_{\mathrm{i}}}, \mathrm{u}_{\mathrm{a}_{\mathrm{j}}}, \mathrm{u}_{\mathrm{a}_{\mathrm{i}} \mathrm{a}_{\mathrm{j}}}^{\prime}\right) \geq 0,\right. \\
\left.(\mathrm{i}, \mathrm{j}) \in \Xi, \widehat{\Phi}_{\mathrm{i}}\left(\mathrm{u}_{\mathrm{a}_{\mathrm{i}}}, \mathrm{p}\right) \geq 0, \mathrm{i} \in \mathrm{I}_{\mathrm{n}}\right\},
\end{gathered}
$$

где $\mathrm{F}(\mathrm{u})=\mathrm{p}, \widehat{\Phi}_{\mathrm{ij}}^{\prime}\left(\mathrm{u}_{\mathrm{a}_{\mathrm{i}}}, \mathrm{u}_{\mathrm{a}_{\mathrm{j}}}, \mathrm{u}_{\mathrm{a}_{\mathrm{i}} \mathrm{a}_{\mathrm{j}}}^{\prime}\right)$ - псевдонормализованная квази рhі-функция определенная в (2), $a_{i}, a_{j} \in I_{N},(i, j) \in \Xi, u_{a_{i} a_{j}}^{\prime}=u_{P}^{s}, s=1, \ldots, m, \Xi$ приведено в (7), для $\mathrm{K}_{\mathrm{i}}$ и $\mathrm{K}_{\mathrm{j}}$, учитывая минимально допустимые расстояния $\rho_{\mathrm{qg}}>0, \widehat{\Phi}_{\mathrm{i}}\left(\mathrm{u}_{\mathrm{a}_{\mathrm{i}}}, \mathrm{p}\right)-$ псев- 
донормализованная рhі-функция, определенная в (6) для $\mathrm{K}_{\mathrm{i}}$ и объекта $\Omega^{*}$, учитывая минимально допустимые расстояния $\rho_{\mathrm{q}}>0$. Если $\rho_{\mathrm{qg}}$ и $\rho_{\mathrm{q}}$ не заданы, то заменяем псевдонормализованные квази phiфункции $\widehat{\Phi}_{\mathrm{ij}}^{\prime}\left(\mathrm{u}_{\mathrm{a}_{\mathrm{i}}}, \mathrm{u}_{\mathrm{a}_{\mathrm{j}}}, \mathrm{u}_{\mathrm{a}_{\mathrm{i}} \mathrm{a}_{\mathrm{j}}}^{\prime}\right)$ на квази рhi-функции $\Phi_{\mathrm{ij}}^{\prime}\left(\mathrm{u}_{\mathrm{a}_{\mathrm{i}}}, \mathrm{u}_{\mathrm{a}_{\mathrm{j}}}, \mathrm{u}_{\mathrm{a}_{\mathrm{i}} \mathrm{a}_{\mathrm{j}}}^{\prime}\right)$, определенные в (1), и псевдонормализованные phi-функции $\widehat{\Phi}_{\mathrm{i}}\left(\mathrm{u}_{\mathrm{a}_{\mathrm{i}}}, \mathrm{p}\right)$ на phiфункции $\Phi_{\mathrm{i}}\left(\mathrm{u}_{\mathrm{a}_{\mathrm{i}}}, \mathrm{p}\right)$, определенные в (5).

Следует отметить, что для того, чтобы избежать избыточности неравенств в ограничениях включения, можно использовать набор псевдонормализованных рhі-функций $\widehat{\Phi}_{\mathrm{q}}^{\mathrm{h}}\left(\mathrm{u}_{\mathrm{q}}, \mathrm{p}\right) \geq 0, \mathrm{q} \in \mathrm{J}_{\mathrm{N}}$, для выпуклой оболочки невыпуклых многогранников $\mathbb{Q}_{q}$, вместо набора псевдонормализованных phiфункций $\widehat{\Phi}_{\mathrm{i}}\left(\mathrm{u}_{\mathrm{a}_{\mathrm{i}}}, \mathrm{p}\right) \geq 0, \quad \mathrm{i} \in \mathrm{I}_{\mathrm{n}}$, для выпуклых многогранников $\mathrm{K}_{\mathrm{i}}, \mathrm{i} \in \mathrm{I}_{\mathrm{n}}$.

Каждое неравенство квази рhі-функции в (9) представлено системой неравенств с дифференцируемыми функциями. Модель (8)-(9) является невыпуклой непрерывной задачей нелинейного программирования и содержит все глобально оптимальные решения. Таким образом, существует возможность непосредственно использовать глобальные NLPсолверы для решения задачи (8)-(9). Однако, на практике математическая модель (8)-(9) содержит большое количество переменных и огромное число неравенств, а именно: $\mathrm{O}\left(n^{2}\right)$ нелинейных неравенств и $\mathrm{O}\left(n^{2}\right)$ переменных с учетом вспомогательных переменных, необходимых для построения квази рhіфункций, где $n$ - число выпуклых многогранников. В этой связи поиск локально оптимального решения становится практически нереальной задачей для существующих современных NLP-солверов, применяя их непосредственно к модели (8)-(9): для $\mathrm{N}>15$, стартуя из случайной точки и для $\mathrm{N}>30$, стартуя из допустимой точки. Для поиска “хорошего" локально оптимального решения за разумное время предлагается стратегия, которая включает быстрый алгоритм поиска допустимой стартовой точки и специальную процедуру локальной оптимизации. В большинстве случаев эта процедура сводит задачу (8)-(9) к последовательности подзадач нелинейного программирования значительно меньшей размерности и со значительно меньшим числом нелинейных неравенств.

\section{Стратегия решения}

Стратегия решения задачи (8)-(9) основана на методе мультистарта и включает в себя такие этапы:

1) формирование множества $\left\{\varsigma^{0}\right\}$ векторов $\varsigma^{0}=\left(\mathrm{p}^{0}, \mathrm{u}_{1}^{0}, \mathrm{u}_{2}^{0}, \ldots, \mathrm{u}_{\mathrm{N}}^{0}\right)$ допустимых параметров раз- мещения многогранников, размещенных в контейнере $\Omega^{0}$ с размерами $\left(\mathrm{p}^{0}\right)$ в задаче (8)-(9). В данном исследовании используется алгоритм, основанный на гомотопических преобразованиях объектов (см., например, [17]);

2) поиск локального минимума функции цели $F(u)$ в задаче (8)-(9), стартуя из каждой точки множества $\left\{c^{0}\right\}$, полученной на первом этапе. Для поиска локального минимума задачи (8)-(9) используется COMPOLY алгоритм, который подробно описан в [17] для случая, когда контейнер имеет форму кубоида;

3) лучший из найденных на втором этапе локальных минимумов выбирается в качестве локально оптимального решения задачи (8)-(9).

Поскольку задача (8)-(9) не может быть решена для $\mathrm{N}>30$ при непосредственном использовании современных NLP-солверов (стартуя из допустимой точки), предлагается специальный алгоритм поиска локального оптимального решения.

COMPOLY алгоритм сводит задачу (8)-(9), которая имеет большое число неравенств и большую размерность $\mathrm{O}\left(n^{2}\right)$ множества допустимых решений $W(9)$, к последовательности подзадач нелинейного программирования с меньшим количеством нелинейных неравенств $\mathrm{O}(n)$ и меньшей размерности $\mathrm{O}(n)$. Ключевая идея этого алгоритма заключается в следующем. Для каждого вектора допустимых параметров размещения размещаемых многогранников, строится фиксированный индивидуальный кубический контейнер для сфер, описанных вокруг соответствующих выпуклых многогранников. Затем каждая из сфер размещается внутри индивидуальных контейнеров. Движение каждой из сфер в фиксированном индивидуальном контейнере описывается системой, состоящей из шести линейных $\varepsilon$ неравенств. Затем формируется подобласть допустимых решений следующим образом: добавляется $\mathrm{O}(n)$ $\varepsilon$-неравенств (для всех сфер) к системе неравенств (9), что позволяет: удалить $\mathrm{O}\left(n^{2}\right)$ phi-неравенств для тех пар многогранников, чьи индивидуальные контейнеры не пересекаются, а также удалить некоторые избыточные неравенства для ограничения включения многогранников в контейнер.

Далее осуществляется поиск локального минимума на подобласти размерности $\mathrm{O}(n)$, каждая из которых описывается $\mathrm{O}(n)$ нелинейными неравенствами; полученный локальный минимум используется в качестве стартовой точки для следующей итерации. На последней итерации получаем локальный минимум задачи (8)-(9).

\section{Вычислительные эксперименты}

Эксперименты проводились на компьютере AMD Athlon 64 X2 5200+. Язык программирования $\mathrm{C}++$, Windows 7. Для решения задач нелинейного 
программирования (при реализации шагов 1-2) использовалась программа IPOPT [15], https://projects.coin-or.org/Ipopt). Исходные данные об объектах приведены в работах $[16,17]$.

Пример 1. Рассматривается набор $\mathrm{n}=40$ выпуклых многогранников. Контейнером является персечение шаров с центрами в точках $(-10,0,0)$ и $(10,0,0)$ и с одинаковыми переменными радиусами $\mathrm{r}$. На рис. 1, а приведено локально-оптимальное размещение заданного набора многогранников в произвольной выпуклой области. Значение функции цели $-\mathrm{F}^{*}=27.624$. Время решения -3730.34 сек.

Пример 2. Рассматривается набор $\mathrm{N}=25$ невыпуклых многогранников. Контейнером является пересечение шаров с центрами в точках $(-10,0,0)$ и $(10,0,0)$ и с одинаковыми переменными радиусами $\mathrm{r}$. На рис. 1, б приведено локально- оптимальное размещение многогранников. Значение функции цели $\mathrm{F}^{*}=\mathrm{r}^{*}=26.376$. Время решения -8658.87 сек.

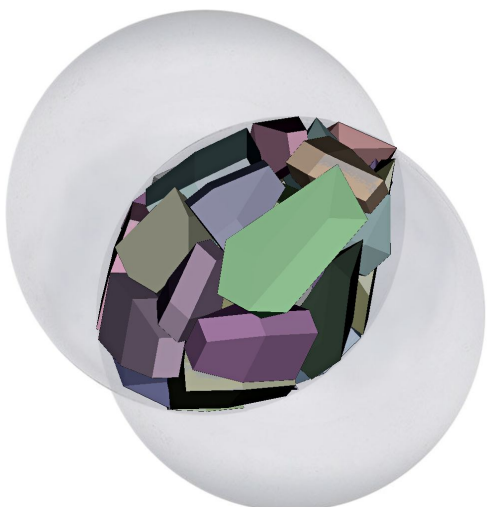

a

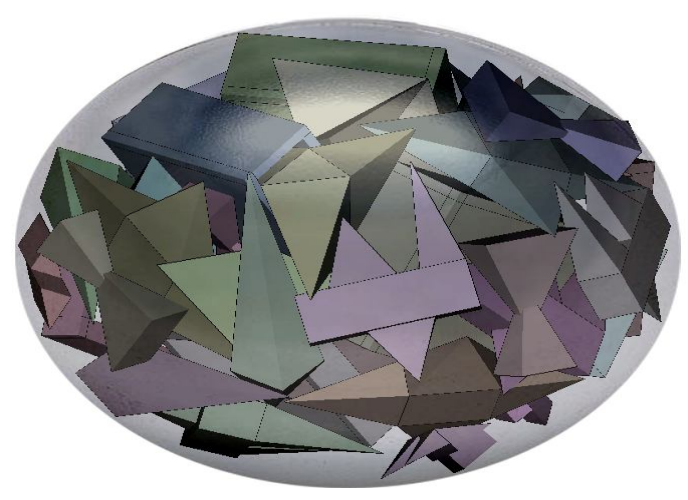

$\Gamma$

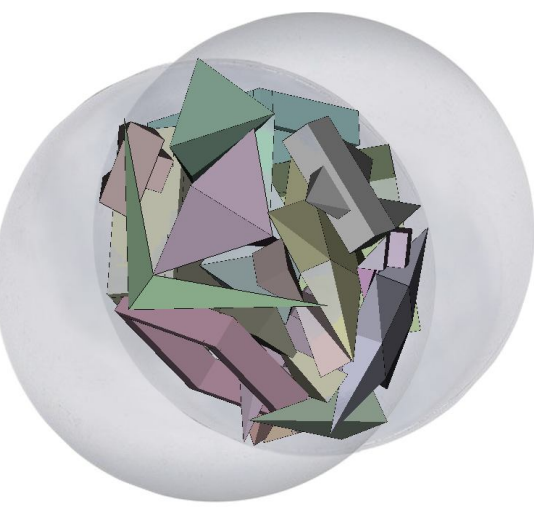

6

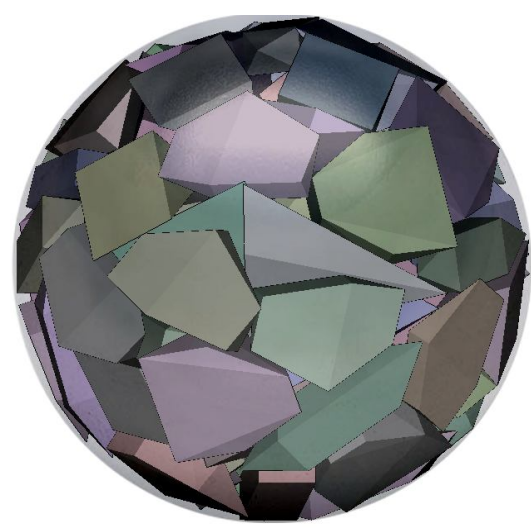

B

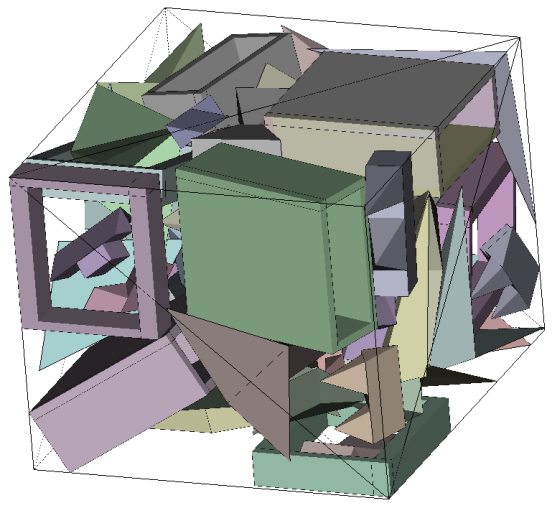

Д

Рис. 2 Локально-оптимальное размещение многогранников для примеров: $\mathrm{a}-1 ;$ б $-2 ;$ в $-5 ;$ г $-3 ;$ д -4

Пример 3. Рассматривается набор $\mathrm{N}=40$ невыпуклых многогранников. На рис. 1, г изображено локально оптимальное размещение многогранников в эллипсоиде, который имеет размеры: $a^{*}=28.3608, b^{*}=24.8157, c^{*}=21.270$. Использовалось 10 стартовых точек. Время решения - 29491.35 сек.

Пример 4. Рассматривается набор $\mathrm{N}=50$ невыпуклых многогранников. На рис. 1, д приведено локально оптимальное размещение набора невыпуклых многогранников. Контейнер имеет следующие размеры и объем $\left(1^{*}, \mathrm{w}^{*}, \mathrm{~h}^{*}\right)=(46.9742, \quad 34.8305, \quad 41.6923)$ и $\mathrm{F}\left(\mathrm{u}^{*}\right)=68214.5610$. Использовалась одна стартовая точка и установлен предел времени 10 часов.
Пример 5. Рассматриваются $N=98$ выпуклых многогранников с заданными весами. На рис. 1, в приведена локально оптимальная балансная упаковка многогранников в шаре минимального радиуса. Цель - минимизация радиуса $r$ с минимальным отклонением $d$ системы от центра шара: $\quad \mathrm{F}\left(\mathrm{u}^{*}\right)=\kappa_{1} \mathrm{r}^{*}+\kappa_{2} \mathrm{~d}^{*}, \quad \mathrm{r}^{*}=18.0409, \quad \mathrm{~d}^{*}=0$. Использовалась одна стартовая точка. Время решения - 26150.23 сек.

Примеры локально оптимальных упаковок многогранников в цилиндрическом и шаровом контейнерах приведены в работе [18].

\section{Выводы}

В статье приведена математическая модель задачи оптимальной упаковки многогранников в вы- 
пуклом контейнере в виде задачи нелинейного программирования.

Предложен эффективный алгоритм решения, который включает в себя быстрый алгоритм поиска стартовой точки, а также процедура COMPOLY локальной оптимизации для поиска “хороших" локально оптимальных решений. Процедура COMPOLY позволяет существенно сократить вычислительные затраты (время и память).

\section{Список литературы}

1. Bennell, J., Oliveira, J. (2008). The geometry of packing problems:A tutorial.European Journal of Operational Research184:397-415.

2. Gomes, A. Miguel Irregular Packing Problems: Industrial Applications and New Directions Using Computational Geometry. (2014) Paper in special issue on "Cutting and Packing". Vol 11 | Part 1, 378-383. DOI: 10.3182/20130522-3-BR-4036.00113.

3. Wascher, G., Hauner, H., Schumann, H. (2007). An improved typology of cutting and packing problems. Eur. J. Oper. Res., 183(3), 1109-1130.

4. Chazelle, B., Edelsbrunner, H., Guibas, L. J. (1989). The complexity of cutting complexes. Discr. \& Comput. Geom., 4(2), 139-181. DOI: 10.1007/BF02187720.

5. Chen, E. R., Klotsa, D., Engel, M., Damasceno, P. F., Glotzer, S. C. (2014). Complexity in surfaces of densest packings for families of polyhedra. Phys Rev X 4(1), DOI:10.1103/PhysRevX.4.011024.

6. Galrão, R. A., Oliveira J. F., Gonçalves J. F., Lopes M. P. (2016) A container loading algorithm with static mechanical equilibrium stability constraints. Transportation Research, (Part B), 91, 565-581. DOI: 10.1016/j.trb.2016.06.003.

7. Smeets, B., Odenthal, T., Vanmaercke, S., Ramon, $H$. (2015). Polygon-based contact description for modeling arbitrary polyhedra in the Discrete Element Method. Computer Methods in Applied Mechanics and Engineering, 290, 277-289. DOI: 10.1016/j.cma.2015.03.004.

8. Tasios, N., Gantapara, A. P., Dijkstra M. (2014). Glassy dynamics of convex polyhedra. The Journal of Chemical Physics. 141: 224502. PMID 25494755 DOI: 10.1063/1.49029922.
9. Egeblad, J., Nielsen, B. K., Brazil, M. (2009). Translational packing of arbitrary polyhedra. Comp. Geom., 42(4), 269-288. DOI:10.1016/j.comgeo.2008.06.003.

10. Fasano, G. A. (2013). Global Optimisation point of view for non-standard packing problems. J. Glob. Optim., 55(2), 279-299. DOI: 10.1007/s10898-012-9865-8.

11. Torquato, S., Jiao, Y. (2009). Dense polyhedral packings: Platonic and Archimedean solids. Phys. Rev., 80, 041104. DOI: 10.1103/PhysRevE.80.041104.

12. Chernov, N., Stoyan, Y., Romanova, T. (2010). Mathematical model and efficient algorithms for object packing problem. Comput. Geom.: Theory and Appl., 43(9), 535-553. DOI:10.1016/j.comgeo.2009.12.003.

13. Fischer, K., Gärtner, B. and Kutz, M. (2003). Fast Smallest-Enclosing-Ball Computation in High Dimensions. Algorithms - ESA 2003, 2832, 630-641. DOI:10.1007/978-3540-39658-1 57.

14. Stoyan, Y., Pankratov, A., Romanova, T. (2016). Quasi phi-functions and optimal packing of ellipses. J. of Glob. Optim., 65 (2), 283-307. DOI: 10.1007/s10898-0150331-2.

15. Wachter, A., Biegler, L. T. (2006). On the implementation of an interior-point filter line-search algorithm for large-scale nonlinear programming. Math. Program., 106 (1), 25-57. DOI: 10.1007/s10107-004-0559-y.

16. Stoyan, Y. G., Gil, N. I., Pankratov, A. V., et al., (2004). Packing Non-convex Polyhedra into a Parallelepiped. Technische Universitat Dresden. Mode of access: http://www.math.tu-dresden.de/ scheith/ABSTRACTS/ PREPRINTS/04-non-conv.pdf

17. Romanova, T., Bennell, J., Stoyan, Y., Pankratov, A. (2018) Packing of concave polyhedra with continuous rotations using nonlinear optimization. European Journal of $\begin{array}{llll}\text { Operational Research } 268 & \text { (1), 37-53. }\end{array}$ https://doi.org/10.1016/j.ejor.2018.01.025

18. Панкратов, А.В., Романова, Т.Е., Стоян, Ю.Е., Чугай, А.М. (2016). Задача оптимизачии упаковки многогранников в сферическом и чилиндрическом контейнерах. Eastern-European J. of Entrepr. Tech., 1/4 (79), 39-47.

\section{Надійшла до редколегї 24.10.2017}

Рецензент: д-р техн. наук, проф. І.В. Гребеннік, Харківський національний університет радіоелектроніки, Харків.

\title{
УПАКОВКА БАГАТОГРАННИКІВ В ОПУКЛИЙ КОНТЕЙНЕР МІНІМАЛЬНОГО ОБСЯГУ
}

\author{
Ю.С. Стоян, А.В. Панкратов, Т.С. Романова
}

Розглядається задача упаковки заданого набору довільних багатогранників в опуклий контейнер мінімального обсягу. Допускаються безперервні обертання і трансляиії багатогранників. Враховуються мінімально допустимі відстані між многогранниками і обмеження балансу. Побудовано математичну модель у вигляді задачі нелінійного програмування з використанням рhі-функцій $і$ квазі рhі-функиій. Запропоновано ефективний метод рішення, який включає в себе швидкий алгоритм пошуку допустимої стартової точки і процедуру локальної оптимізації. Перевага запропонованого методу підтверджується результатами численних експериментів.

Ключові слова: упаковка, багатогранники, безперервне обертання, опуклий контейнер, математичне моделювання, нелінійна оптимізаиія.

\section{PACKAGING POLYHEDRONS INTO A CONVENIENT CONTAINER OF MINIMUM VOLUME}

\section{Yu.E. Stoyan, A.V. Pankratov, T.Ye. Romanova}

The problem of packing a given set of arbitrary polyhedron into a convex container of minimal volume is considered. Continuous rotations and translations of polyhedron are allowed. Minimum distances between polyhedron and balance constraints are taken into account. A mathematical model is constructed in the form of a non-linear programming problem using phifunctions and quasi-phi-functions. An effective method of solution is proposed that includes a fast algorithm for finding an acceptable starting point and a procedure for local optimization. The advantage of the proposed method is confirmed by the results of numerical experiments.

Keywords: packing, polyhedron, continuous rotation, convex container, mathematical modeling, nonlinear optimization. 